\title{
Temperaturabhängiges Anzieh- und Löseverhalten von Schraubenverbindungen mit Zinklamellenüberzügen
}

\author{
A. Eberhard ${ }^{1,2}$ (D) M. Stähler ${ }^{3} \cdot$ S. Beyer ${ }^{1} \cdot$ M. Klein² ${ }^{2}$ (D) M. Oechsner² \\ Eingegangen: 28. Juni 2019 / Angenommen: 2. Dezember 2020 / Online publiziert: 31. März 2021 \\ (c) Der/die Autor(en) 2021
}

\section{Zusammenfassung}

In diesem Beitrag werden die Ergebnisse von Warmlöseversuchen nach VDA 235-203/VW 01131 mit denen von Anziehund Löseversuchen an einem Anziehprüfstand verglichen. Die Versuche werden bei Temperaturen von Raumtemperatur (RT) bis $\vartheta=150^{\circ} \mathrm{C}$ bei Variation der Schraubenabmessung (M6 und M10), der Schraubenkopfgeometrie, des Beschichtungssystems von Schraube (Zinklamelle und Zinknickel sowie mit und ohne Decksicht) und Mutter (blank, verzinkt) sowie des Typs der Gegenlage (Scheibe des Typs HL und KTL (Kathodische Tauchlackierung)-Stahlleiste) durchgeführt. Die Ergebnisse werden im Hinblick auf die unterschiedlichen Prüfmethoden vergleichend bewertet.

\section{Temperature-dependent tightening and loosening behavior of bolted joints with zinc flake coatings}

\begin{abstract}
The results of hot-loosening tests according to VDA 235-203/VW 01131 and tightening and loosening tests on a tightening test rig are compared in this paper. The experiments are carried out from room temperature up to $\vartheta=150^{\circ} \mathrm{C}$ with variation of the dimension (M6 and M10), the bolt head geometry, the coating system of the bolt (zinc flake and zinc nickel as well as with and without top coat), the coating of the nut (bare, galvanized) and the type of counterpart (washer type HL and KTL (cathodic dip coating) steel bar).
\end{abstract}

$\triangle$ A. Eberhard

eberhard@mpa-ifw.tu-darmstadt.de

M. Stähler

MStaehler@doerken.de

S. Beyer

sbeyer@schraubenverband.de

M. Klein

m.klein@mpa-ifw.tu-darmstadt.de

M. Oechsner

oechsner@mpa-ifw.tu-darmstadt.de

1 Deutscher Schraubenverband e. V., Goldene

Pforte 1, 58093 Hagen, Deutschland

2 Zentrum für Konstruktionswerkstoffe (MPA/IfW), Technische Universität Darmstadt, Grafenstraße 2, 64283 Darmstadt, Deutschland

3 Dörken Coatings GmbH \& Co. KG, Wetterstraße 58, 58313 Herdecke, Deutschland

\begin{tabular}{ll}
\multicolumn{2}{l}{ Symbolverzeichnis } \\
$\mathrm{d}_{2}[\mathrm{~mm}]$ & Flankendurchmesser \\
$\mathrm{l}_{\mathrm{K}}[\mathrm{mm}]$ & Klemmlänge \\
$\mathrm{n}\left[\mathrm{min}^{-1}\right]$ & Drehzahl \\
$\mathrm{t}[\mathrm{s}]$ & Zeit \\
$\mathrm{D}_{\mathrm{b}}[\mathrm{mm}]$ & Reibdurchmesser \\
$\mathrm{F}_{\mathrm{V}}[\mathrm{kN}]$ & Vorspannkraft \\
$\Delta \mathrm{F}_{\mathrm{Z}}[\mathrm{kN}]$ & Vorspannkraftverlust infolge Setzens \\
$\mathrm{N}[-]$ & Versuchsanzahl \\
$\mathrm{P}[\mathrm{mm}]$ & Steigung \\
$\mathrm{T}[\mathrm{Nm}]$ & Anziehdrehmoment \\
$\mathrm{T}_{\mathrm{LG}}[\mathrm{Nm}]$ & Losdrehmoment \\
$\mathrm{T}_{\mathrm{LH}}[\mathrm{Nm}]$ & Losbrechmoment \\
$\vartheta\left[{ }^{\circ} \mathrm{C}\right]$ & Temperatur \\
$\mu_{\mathrm{H}}[-]$ & Haftreibungszahl \\
$\mu_{\mathrm{tot}}[-]$ & Gesamtreibungszahl \\
$\mu_{\mathrm{totA}}[-]$ & Gesamtreibungszahl beim Anziehen \\
$\mu_{\text {totL }}[-]$ & Gesamtreibungszahl beim Lösen \\
$\varphi\left[{ }^{\circ}\right]$ & Steigungswinkel \\
$\varnothing$ & Durchschnitt
\end{tabular}




\section{Einleitung}

Schraubenverbindungen sind eine der wichtigsten lösbaren Verbindungen von zwei oder mehreren Bauteilen und haben damit eine sehr große Verbreitung und Bedeutung in fast allen Bereichen des konstruktiven Ingenieurwesens. Die prozess- und betriebssichere Auslegung von Schraubenverbindungen ist zur Vermeidung von Schadensfällen (Dauerbruch oder selbsttätiges Losdrehen) von entscheidender Bedeutung. Hierzu ist es notwendig, dass bei der Auslegung definierte Montagebedingungen festgelegt werden, um die erforderliche Vorspannkraft $\mathrm{F}_{\mathrm{V}}$ sicher zu erreichen. Die Schraubenverbindung muss später den Betriebsund Umgebungsbedingungen standhalten, auch wenn die Betriebseinflüsse bei der Auslegung nicht vollständig bekannt sind. In diesem Zusammenhang kommt der gewählten Beschichtung bzw. dem Beschichtungssystem von Schraube und Mutter eine große Bedeutung zu. Neben einem ausreichenden Korrosionsschutz wird im deutschen Automobilbereich üblicherweise eine geringe Gesamtreibungszahlspanne von $\mu_{\mathrm{tot}}=0,09$ bis 0,14 [1] beim Anziehvorgang zugelassen, um die Streuung der Vorspannkraft $F_{V}$ ebenfalls gering zu halten. Geringfügige Abweichungen von dieser Gesamtreibungszahlspanne sind in Werksnormen wie [2, 3] und [4] zu finden. Weiterhin soll bei temperaturbelasteten Schraubenverbindungen kein temperaturabhängiges selbsttätiges Losdrehen auftreten. Hierzu wird in [5] für eine Temperatur von $\vartheta=150^{\circ} \mathrm{C}$ ein Grenzwert der Gesamtreibungszahl für die Selbsthemmungsgrenze von $\mu_{\mathrm{tot}}=0,06$ angegeben.

\section{Die Schraubenverbindung als tribologisches System}

Reibung und Verschleiß können bei einer Schraubenverbindung als tribologisches System dargestellt werden (Abb. 1). Die Struktur des Systems wird aus dem Grundkörper wie Schraube und Mutter, dem Gegenkörper wie verspannte Bauteile, Scheiben unterschiedlicher Härte oder Leisten und dem Zwischenstoff sowie dem Umgebungsmedium gebildet. Der Zwischenstoff wird durch eine Beschichtung der Verbindung, ein zusätzliches Schmiermittel oder gegebenenfalls durch die Rückstände aus einer vorherigen Reinigung des Gegenkörpers bestimmt.

Auf die Struktur der Schraubenverbindung wirkt ein Beanspruchungskollektiv, das sich aus Belastung, Bewegung und Temperatur zusammensetzt. Beim Anziehvorgang führt ein steigendes Anziehdrehmoment $\mathrm{T}$ zu einer steigenden Vorspannkraft $F_{V}$ in der Schraube, die im Betrieb durch axiale oder exzentrische Kräfte, Querkräfte, Momente oder eine kombinierte Belastung überlagert werden kann. Eine zusätzliche Beeinflussung entsteht durch Unterschiede bei den Montage- und Betriebstemperaturen.

Bei Variation von Grundkörper, Gegenkörper, Zwischenstoff/Umgebungsmedium, Belastung, Bewegung und Temperatur können sich die Reibungszahlen und mit ihnen zentrale Eigenschaften der Schraubenverbindung ändern. Das tribologische System der Schraubenverbindung hat einen signifikanten Einfluss auf die Ergebnisse von Schwingversuchen nach DIN 969 [7], Vibrationsversuchen nach DIN 65151 [8], Anziehversuchen nach DIN EN ISO 16047 [9] oder Warmlöseversuchen nach VDA 235-203 [10]/VW 01131 [5].
Abb. 1 Allgemeines tribologisches System [nach 6], angewendet auf Schraubenverbindungen

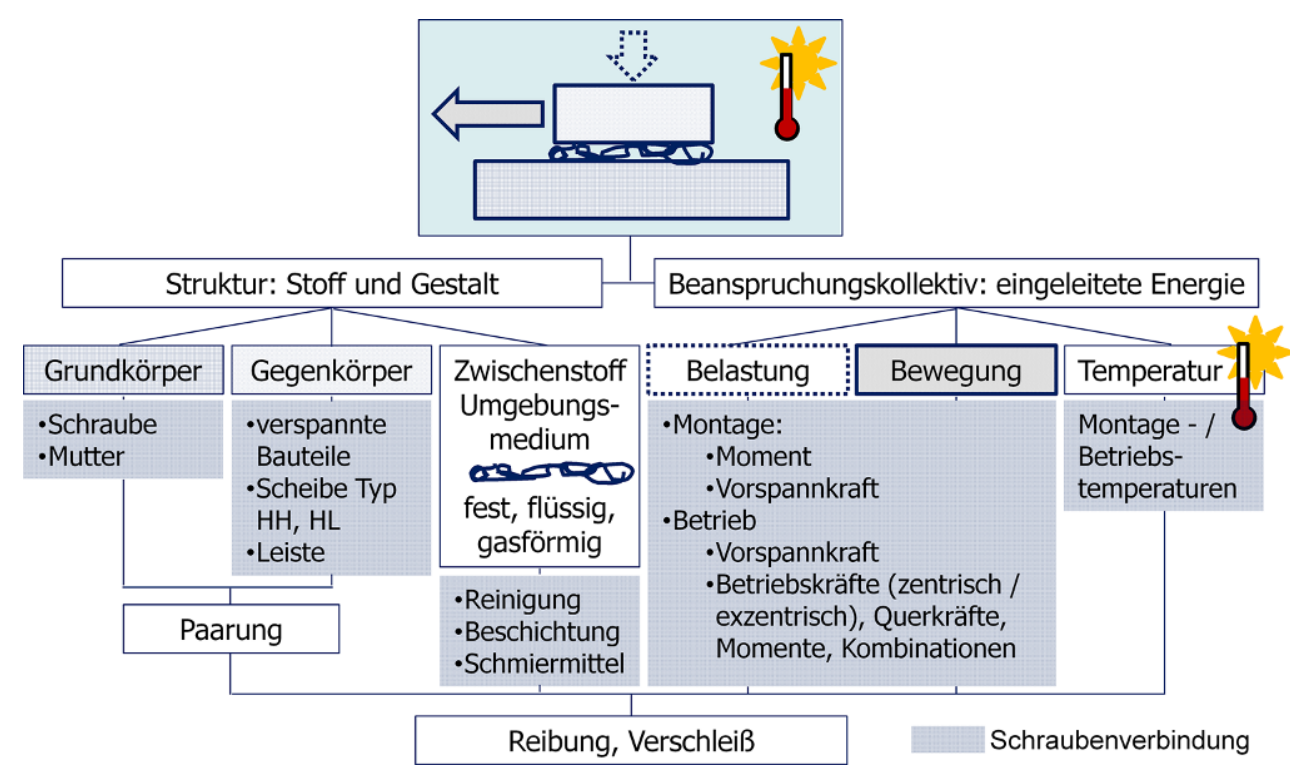


Tab. 1 Versuchsmatrix zur vergleichenden Untersuchung der Prüfgutvarianten P1-P5 mit zwei Prüfmethoden bezüglich der Temperaturabhängigkeit auf die Gesamtreibungszahl $\mu_{\text {tot }}$ und das Verhältnis von Losdreh- zu Anziehdrehmoment $\mathrm{T}_{\mathrm{LG}} / \mathrm{T}$

\begin{tabular}{|c|c|c|c|c|}
\hline \multicolumn{3}{|c|}{ Prüfgutvariante } & \multicolumn{2}{|c|}{ Prüfmethode mit Versuchsanzahl $N$} \\
\hline & Schrauben, Muttern und Gegenlagen & Beschichtung & $\begin{array}{l}1 \\
\text { Anziehprüfstand }\end{array}$ & $\begin{array}{l}2 \\
\text { Warmlöseversuche nach VDA 235-203 } \\
{[10] / \text { VW } 01131[5]}\end{array}$ \\
\hline \multirow[t]{2}{*}{$\overline{\mathrm{P} 1}$} & Kombischrauben M6×55-8,8 & B1-B4 & - & $N=10$ \\
\hline & Muttern M6-10 [11] & Blank & & \\
\hline \multirow[t]{2}{*}{$\mathrm{P} 2$} & Kombischrauben M6×55-8,8 & B2 & - & $N=5$ \\
\hline & Muttern M6-10 [12] & Verzinkt & & \\
\hline \multirow[t]{3}{*}{ P3 } & $\begin{array}{l}\text { Schrauben M6×55-8,8; außentragend } \\
\text { [13] }\end{array}$ & B2 & - & $N=5$ \\
\hline & Muttern M6-10 [12] & Verzinkt & & \\
\hline & Scheiben Typ HL [9] & Blank & & \\
\hline \multirow[t]{3}{*}{ P4 } & $\begin{array}{l}\text { Schrauben M6×55-8,8; außentragend } \\
\text { [13] }\end{array}$ & B2 & - & $N=5$ \\
\hline & Muttern M6-10 [11] & Blank & & \\
\hline & Scheiben Typs HL [9] & Blank & & \\
\hline \multirow[t]{4}{*}{ P5 } & Flanschschrauben M10×65-10,9 & B1-B4 & $N=5$ bis 10 & $N=10$ \\
\hline & Muttern M10-10 [11] & Blank & & \\
\hline & Scheiben Typs HL [9] & Blank & & \\
\hline & Stahlleiste & KTL & - & \\
\hline
\end{tabular}

\section{Prüfgut}

Die Übersicht des Prüfguts ist in Tab. 1 gelistet. Für den Vergleich der beiden Prüfmethoden zur Untersuchung der Temperaturabhängigkeit auf die Gesamtreibungszahl $\mu_{\text {tot }}$ und des Verhältnisses von Losdreh- zu Anziehdrehmoment $\mathrm{T}_{\mathrm{LG}} / \mathrm{T}$ wurde Prüfgutvariante $\mathrm{P} 5$ herangezogen, die mit den folgenden Beschichtungen vorliegt:

- Zinklamelle

- nur Basisschicht (B1)

- Basisschicht und organische Deckschicht mit integriertem Schmiermittel (B2)

- Basisschicht und silikatische Deckschicht mit integriertem Schmiermittel (B3)

- Zinknickel

- Basisschicht (passiviert) und anorganische Versiegelung mit integriertem, organischem Schmiermittel (B4)

In den Warmlöseversuchen nach VDA 235-203 [10]/VW 01131 [5] werden zusätzlich der Einfluss der Schraubenabmessung, der Schraubenkopfauflage, der Gegenlage und der Mutternbeschichtung betrachtet (Prüfgutvarianten P1-P5). Hierbei liegen die Prüfgutvarianten P2-P4 nur in der Zinklamellenbeschichtung B2 vor.

Um einen Einfluss der Reinigung der Referenzteile auf die Ergebnisse auszuschließen, werden die Muttern und die Scheiben des Typs HL [9] zentral gereinigt und vor dem Versand luftdicht eingeschweißt. Des Weiteren kommen die
Schrauben jeweils aus einem Fertigungs- und Beschichtungslos.

\section{Anzieh- und Löseversuche an einem Anziehprüfstand}

An einem Anziehprüfstand der Fa. Schatz AG (Klasse 1) wurden mit Prüfgutvariante P5 Anzieh- und Löseversuche bei unterschiedlichen Temperaturen (RT, $\vartheta=80^{\circ} \mathrm{C}$ und $\vartheta=150^{\circ} \mathrm{C}$ ) durchgeführt. Bei diesem Prüfstand befinden sich der Mehrkomponenten-Messwertaufnehmer (Vorspannkraft und Gewindemoment) in einer Wärmekammer und der Motor mit Antrieb und Drehmomentaufnehmer außerhalb der Wärmekammer. Der temperaturkompensierte Mehrkomponenten-Messwertaufnehmer wird somit für die Versuche unter Temperatur zusammen mit der Wärmekammer aufgeheizt. Das Erreichen der Temperatur in der Schraubenverbindung wurde zu Beginn der Versuchsreihen mit einem Temperaturmessgerät Typ 735-2 der Fa. Testo AG (erweiterte Messunsicherheit von $\pm 1,5^{\circ} \mathrm{C}$ ) überprüft.

Beim Anziehvorgang wurde mit einem Anziehdrehmoment von $\mathrm{T}=55 \mathrm{Nm}$ und einer Drehzahl von $n=20 \mathrm{~min}^{-1}$ montiert. Anschließend folgte eine Wartezeit in Abhängigkeit der Temperatur. Die Verbindung wurde schließlich mit einer Drehzahl von $n=5 \mathrm{~min}^{-1}$ wieder gelöst. Jede Schraube, Mutter und Scheibe des Typs HL wurde nur einmal verwendet. Ausgewertet wurden die Gesamtreibungszahlen $\mu_{\text {tot }}$ bei einer Vorspannkraft von 
Abb. 2 Gesamtreibungszahlen $\mu_{\text {tot }}$ (Gleiten) der Anziehund Löseversuche, ermittelt an einem Anziehprüfstand mit Schrauben der Abmessung M10 (Prüfgutvariante P5)
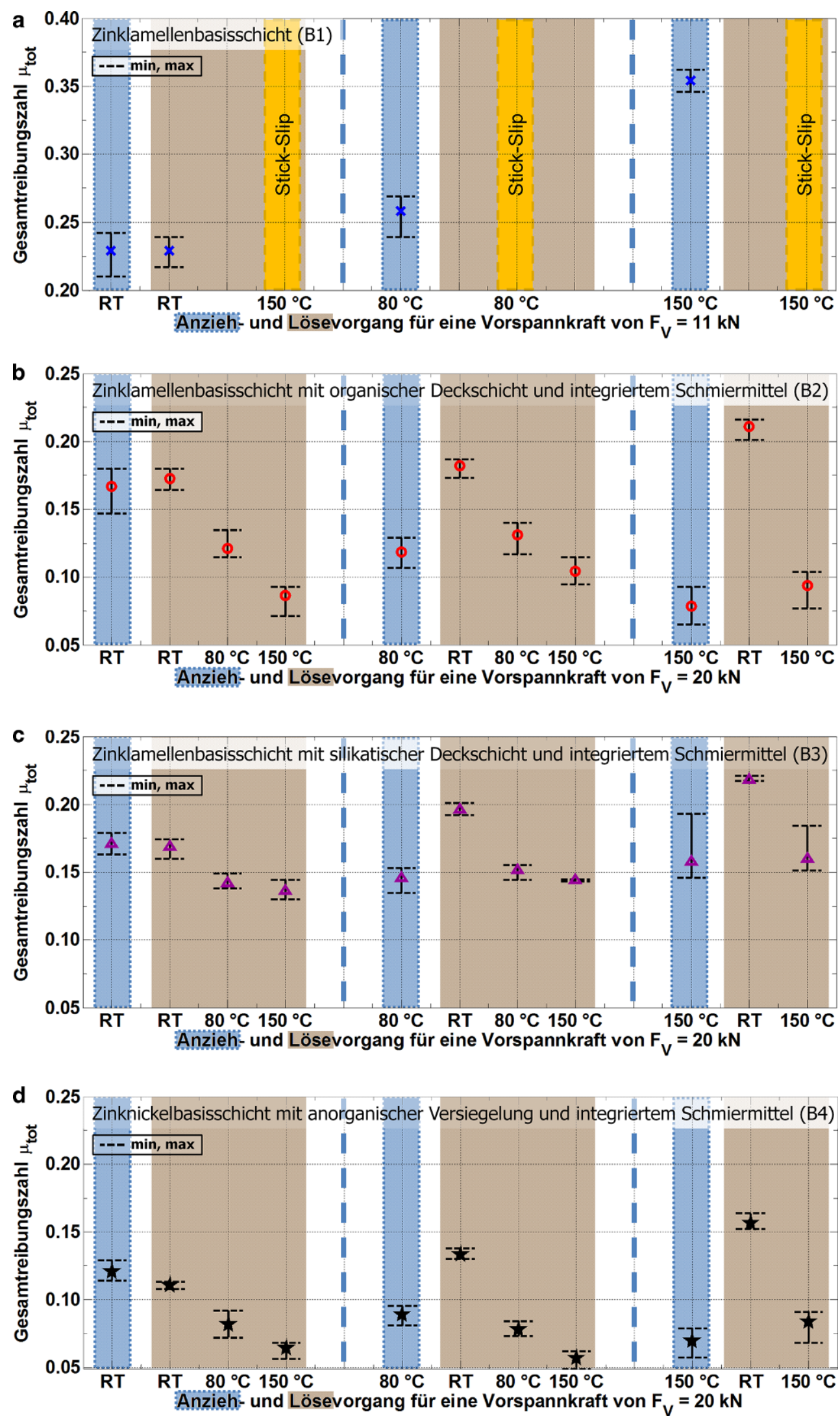
- $F_{\mathrm{V}}=11 \mathrm{kN}$ für Schrauben mit Zinklamellenbeschichtung B1

- $F_{\mathrm{V}}=20 \mathrm{kN}$ für Schrauben mit Zinklamellenbeschichtung B2 und B3 sowie Zinknickelbeschichtung B4.

Während der Aufheizzeit von RT auf $\vartheta=80^{\circ} \mathrm{C}(\mathrm{t}=2,5 \mathrm{~h})$ bzw. auf $\vartheta=150^{\circ} \mathrm{C}(\mathrm{t}=4 \mathrm{~h})$ kommt es unabhängig von der Beschichtung zunächst zu einem Setzen der Verbindung. Hierbei stellt sich ein Vorspannkraftverlust infolge Setzens von durchschnittlich $\varnothing \mathrm{F}_{\mathrm{Z}}=0,5 \mathrm{kN}$ (B2 und B3) bzw. $0,7 \mathrm{kN}$ (B4) ein. Der Mehrkomponenten-Messwertaufnehmer dehnt sich beim Aufheizen in der Wärmekammer auf $\vartheta=80^{\circ} \mathrm{C}$ stärker aus als die Schraube, so dass sich der Vorspannkraftverlust $\mathrm{F}_{\mathrm{Z}}$ infolge Setzens für die Prüfgutvariante P5 mit den Beschichtungen B2, B3 und B4 am Ende der Aufheizzeit aufhebt und sich gemittelt die gleiche Vorspannkraft einstellt, wie unmittelbar nach dem Anziehen. Hingegen kommt es während der Aufheizphase von RT auf $\vartheta=150^{\circ} \mathrm{C}$ bei den Beschichtungen B2, B3 und B4 nach dem Setzvorgang und dem Vorspannkraftanstieg zu einer temperaturbedingten Vorspannkraftrelaxation. Direkt vor dem Lösevorgang liegt ein Vorspannkraftverlust von gemittelt $1 \mathrm{kN}$ vor (ca. $4 \%$ für B2 und B3 sowie 3\% für B4).

Bei der Zinklamellenbeschichtung B3 zeigen sich beim Anzieh- und Lösevorgang für identische Temperaturen im Mittel ähnliche Gesamtreibungszahlen $\varnothing \mu_{\text {tot }}$ (Abb. 2). Für die Zinklamellenbeschichtung B2 ergeben sich geringfügig höhere gemittelte Gesamtreibungszahlen $\varnothing \mu_{\text {tot }}$ bei den Lösevorgängen, verglichen mit den Anziehvorgängen bei gleichen Temperaturen.

Dieses Verhalten von ähnlichen gemittelten Gesamtreibungszahlen $\varnothing \mu_{\text {tot }}$ beim Anzieh- und Lösevorgang ergibt sich auch bei RT für Schrauben mit der Zinklamellenbeschichtung B1. Jedoch können bei dieser Beschichtungsvariante für höhere Temperaturen $\left(\vartheta=80^{\circ} \mathrm{C}\right.$ und $\left.\vartheta=150^{\circ} \mathrm{C}\right)$, die konstant über den Versuch gehalten werden, keine Gesamtreibungszahlen beim Lösevorgang $\mu_{\text {totL }}$ berechnet werden, da es zu Stick-Slip-Effekten kommt. Das Auftreten von Stick-Slip kann unter anderem von der Drehzahl n, der Verlängerung zwischen dem Antrieb und dem Steckschlüsseleinsatz am Schraubenkopf sowie von der Beschichtung abhängig sein [14]. Da die Versuche in Anlehnung an VW 01131 [5] durchgeführt wurden, konnte die Drehzahl $n$ nicht variiert werden. Auf Grund der Konstruktion der Wärmekammer war es auch nicht möglich, die Verlängerung zwischen dem Antrieb und dem Steckschlüsseleinsatz am Schraubenkopf zu verringern.

Mit steigender Temperatur beim Anziehvorgang ergibt sich eine Erhöhung der gemittelten Gesamtreibungszahlen $\varnothing \mu_{\text {tot }}$ bei Schrauben, die nur eine Basisschicht (B1) aufweisen $\left(\varnothing \mu_{\mathrm{totA}} \mathrm{RT}_{\mathrm{RT}}=0,23 ; \varnothing \mu_{\mathrm{totA} 80^{\circ} \mathrm{C}}=0,26\right.$ und $\left.\varnothing \mu_{\text {totA } 150^{\circ} \mathrm{C}}=0,35\right)$. Hingegen fallen bei Schrauben mit
Zinklamellenbeschichtung B2 die gemittelten Gesamtreibungszahlen stärker ab $\left(\varnothing \mu_{\mathrm{totA} \mathrm{RT}}=0,17 ; \varnothing \mu_{\mathrm{totA} 80^{\circ} \mathrm{C}}=0,12\right.$ und $\left.\varnothing \mu_{\mathrm{totA} 150^{\circ} \mathrm{C}}=0,08\right)$ als bei Schrauben mit Zinklamellenbeschichtung B3 $\left(\varnothing \mu_{\mathrm{totA} \text { RT }}=0,17 ; \varnothing \mu_{\mathrm{totA} 80^{\circ} \mathrm{C}}=0,15\right.$ und $\left.\varnothing \mu_{\mathrm{totA} 150^{\circ} \mathrm{C}}=0,16\right)$.

Für den Lösevorgang von Schrauben mit Zinklamellenbeschichtung B3 ergibt sich weiterhin, dass die gemittelte Gesamtreibungszahl für Versuche mit Anziehen bei RT und Lösen bei einer Temperatur von $\vartheta=80^{\circ} \mathrm{C}$ um $17 \%$ abfällt. Bei Versuchen mit Anziehen bei RT und Lösen bei $\vartheta=150{ }^{\circ} \mathrm{C}$ konnte lediglich ein Abfall um weitere $3 \%$ ermittelt werden. Somit zeigt die Beschichtung (B3) bereits eine Temperatursensitivität für die Gesamtreibungszahl beim Lösevorgang in einem Bereich, der laut VDA 235-203 [10] definierten Standardtemperaturen von bis zu $\vartheta=90^{\circ} \mathrm{C}$.

Bei den Versuchen mit Zinklamellenbeschichtung B2 kommt es beim Anziehen bei RT und Lösen bei $\vartheta=80^{\circ} \mathrm{C}$ zu einem Abfall der gemittelten Gesamtreibungszahlen $\varnothing \mu_{\text {tot }}$ von $27 \%$. Dieser Abfall nimmt weiter zu, wenn erst bei $\vartheta=150{ }^{\circ} \mathrm{C}$ gelöst wird $(48 \%)$. Somit können diese Untersuchungen die hohe Temperaturempfindlichkeit einer organischen Beschichtung bestätigen [10] und quantifizieren.

Dass die Temperaturabhängigkeit nicht nur bei den hier exemplarisch verwendeten Zinklamellenbeschichtungen (B1, B2 und B3) besteht, kann durch die beispielhaft verwendete Variante der Zinknickelbeschichtung B4 verdeutlicht werden. So fällt die Gesamtreibungszahl bei Versuchen mit Anziehen bei RT und Lösen bei $\vartheta=80^{\circ} \mathrm{C}$ um $32 \%$ sowie Lösen bei $\vartheta=150{ }^{\circ} \mathrm{C}$ um $47 \%$ ab. Dadurch liegen bei dieser Versuchsreihe mit Anziehen bei RT und Lösen bei $\vartheta=150^{\circ} \mathrm{C}$ nicht alle Ergebnisse über der in [5] definierten Selbsthemmungsgrenze von $\mu_{\mathrm{tot}}=0,06$.

\section{Warmlöseversuche nach VDA 235-203/VW 01131}

Zur Untersuchung des Temperaturverhaltens von Beschichtungen auf Schrauben kann der genormte Warmlöseversuch nach VDA 235-203 [10]/VW 01131 [5] angewendet werden. In diesem Versuch wird die Schraubenverbindung in einem Prüfkörper verschraubt und später nach Temperaturbelastung wieder gelöst. Hierbei ist die tatsächliche Vorspannkraft $F_{V}$ des Systems nicht bekannt. Mittels der gemessenen Größe Anziehdrehmoment T, dem ggf. graphisch ermittelten Losdrehmoment $\mathrm{T}_{\mathrm{LG}}[5,10]$ und einem geschätzten prozentualen Vorspannkraftverlust durch Setzen wird auf eine Gesamtreibungszahl $\mu_{\text {tot }}$ beim Gleiten geschlossen.

Bei den hier durchgeführten Versuchen mit den Prüfgutvarianten P1-P5 wurden die Schrauben der Abmessung M6 mit einem Anziehdrehmoment von $\mathrm{T}=8 \mathrm{Nm}$ und die Schraubenabmessung M10 mit $\mathrm{T}=55 \mathrm{Nm}$ jeweils bei RT 
Abb. 3 Verhältnis von Losdrehzu Anziehdrehmoment T $\mathrm{LG} / \mathrm{T}$ über der Gesamtreibungszahl $\mu_{\text {tot }}$ beim Anzieh- $(\mathrm{RT})$ und Lösevorgang $\left(\vartheta=150^{\circ} \mathrm{C}\right)$ für Schrauben der Abmessung M6 (Prüfgutvariante P1, links) und M10 (Prüfgutvariante P5, rechts)
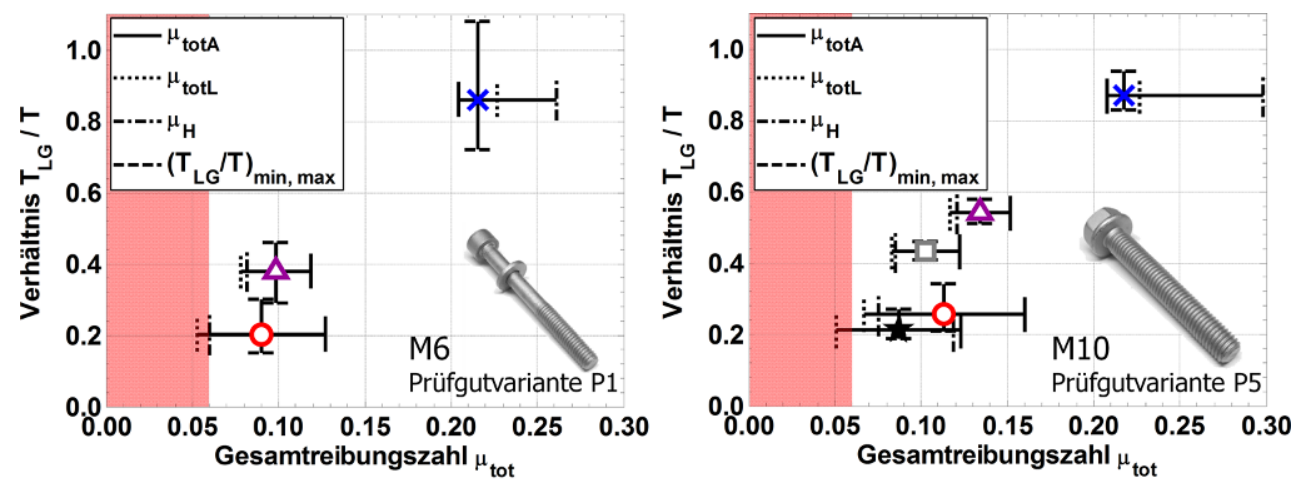

Zinklamellenbeschichtung mit: $\times$ Basisschicht (B1), O Basisschicht und organischer Deckschicht mit integriertem Schmiermittel (B2), $\triangle$ Basisschicht und silikatischer Deckschicht mit integriertem Schmiermittel (B3), $\square$ Basisschicht und silikatischer Deckschicht mit integriertem Schmiermittel (B3) auf KTL

Zinknickelbeschichtung mit: $\star$ Basisschicht (passiviert) und anorganische Versiegelung mit integriertem organischem Schmiermittel (B4) sowie einer Drehzahl von $n=20 \mathrm{~min}^{-1}$ angezogen. Die Wartezeit bis zum Erreichen einer Temperatur von $\vartheta=150^{\circ} \mathrm{C}$ betrug 1,5h (M6) bzw. 2,5 h (M10). Gelöst wurde die Verbindung mit einer Drehzahl von $n=5 \mathrm{~min}^{-1}$. Für die Vorspannkraft wurde ein Setzbetrag von 5\%, wie in VW 01131 [5] definiert, angenommen.

Für die Zinklamellenbeschichtung B1 ergeben sich für die Abmessungen M6 und M10 ein ähnliches gemitteltes Verhältnis von Losdreh- zu Anziehdrehmoment $\mathrm{T}_{\mathrm{GL}} / \mathrm{T}$ sowie ähnliche gemittelte Gesamtreibungszahlen $\mu_{\text {tot }}$ beim Anzieh- und Lösevorgang (Abb. 3). Bei allen anderen untersuchten Zinklamellenbeschichtungen (B2 und B3) liegen stets das Momentenverhältnis $\mathrm{T}_{\mathrm{GL}} / \mathrm{T}$ sowie die Gesamtreibungszahlen $\mu_{\text {tot }}$ der Schraubenabmessung M10 über denen der Abmessung M6. Bei der Zinklamellenbeschichtung B2 für die Schraubenabmessung M6 und bei der Zinknickel-

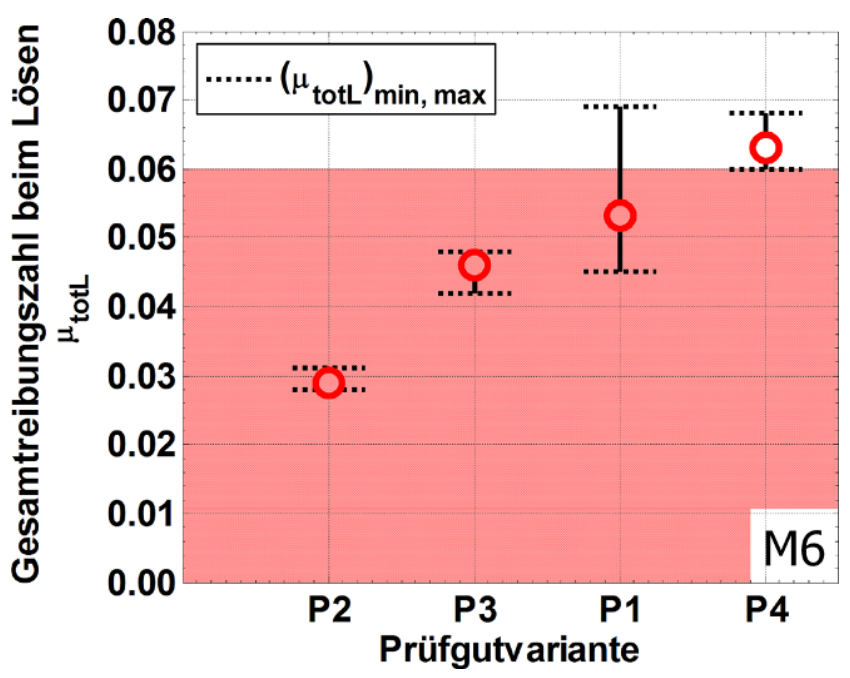

Abb. 4 Gesamtreibungszahlen beim Lösevorgang $\mu_{\text {totL }}$ für eine Temperatur von $\vartheta=150^{\circ} \mathrm{C}$ bei Schrauben der Abmessung M6, beschichtet mit Zinklamellenbasisschicht, organischer Decksicht und integriertem Schmiermittel (B2) für Prüfgutvariante P1-P4 [15] beschichtung B4 der Schraubenabmessung M10 wird sogar die für alle Schraubenabmessungen festgelegte Selbsthemmungsgrenze von $\mu_{\mathrm{tot}}=0,06$ unterschritten [5].

Gründe für diese Unterschiede können allgemein in der Geometrie (Steigung P, Flankendurchmesser $\mathrm{d}_{2}$, Auflagereibdurchmesser $D_{b}$ ), der maximalen Vorspannkraft (Flächenpressung) oder der Komponenten an sich liegen (Abschn. 2), auch wenn bei der Ermittlung der Reibungszahlen die Geometriegrößen berücksichtigt werden. Im Allgemeinen ist der Steigungswinkel für eine Schraube der Abmessung M6 mit Regelgewinde $\left(\varphi=3,40^{\circ}\right)$ größer als bei einer Schraube der Abmessung M10 mit Regelgewinde $\left(\varphi=3,03{ }^{\circ}\right)$. Ein größerer Steigungswinkel $\varphi$ bedeutet für die entsprechende Abmessung eine höhere Selbsthemmungsgrenze des Gewindes $\left(\mu_{\mathrm{M} 6}=0,052, \mu_{\mathrm{M} 10}=0,046\right)$. Somit sind Schrauben der Abmessung M6 stets losdrehgefährdeter als Schrauben der Abmessung M10. Zusätzlich liegt die Schraube der Abmessung M6 als Kombischraube vor (Schraube mit verliersicher aufgerollter Scheibe). Das heißt, dass zum einen die Schraubenkopfauflage und zum anderen auch die Scheibe beschichtet sind. Bei einer solchen Paarung ist üblicherweise die Auflagereibung kleiner als bei (üblicher) nur einseitiger Beschichtung. Weiterhin kann es hier in Abhängigkeit der Scheibengeometrie während der Montage zu einem Wechsel der Relativbewegungen zwischen Schraubenkopfauflage, Scheibe und zu verspannendem Bauteil kommen. Bei der Flanschschraube der Abmessung M10 kommt es stets zu einer Relativbewegung zwischen der beschichteten Schraubenkopfauflage und der unbeschichteten Scheibe des Typs HL, da die Scheibe über eine Nut gegen Verdrehen gesichert ist. 
Abb. 5 Vergleich des Verhältnisses von Losdreh- zu Anziehdrehmoment $\mathrm{T}_{\mathrm{LG}} / \mathrm{T}$ sowie der Gesamtreibungszahl beim Lösevorgang $\mu_{\text {totL }}$ für eine Temperatur von $\vartheta=150^{\circ} \mathrm{C}$ in $\mathrm{Ab}$ hängigkeit der Prüfmethoden (Prüfgutvariante P5), Schraubenabmessung M10

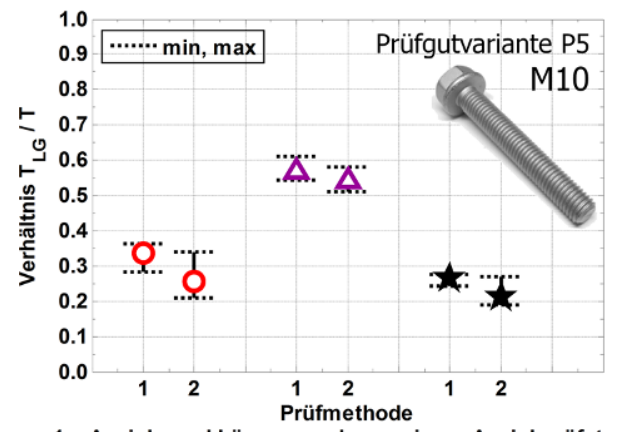

1 = Anzieh- und Löseversuche an einem Anziehprüfstand 2 = Warmlöseversuche nach VDA 235-203 / VW 01131

Zinklamellenbeschichtung mit: O Basisschicht und organischer Deckschicht mit integriertem Schmiermittel (B2), $\triangle$ Basisschicht und silikatischer Deckschicht mit integriertem Schmiermittel (B3)

Zinknickelbeschichtung mit: $\star$ Basisschicht (passiviert) und anorganische Versiegelung mit integriertem organischem Schmiermittel (B4)
Ein Vergleich der Haftreibungszahlen $\mu_{\mathrm{H}}$ beim Lösevorgang $^{1}$ verdeutlicht, dass diese für die Zinklamellenbeschichtungen $\mathrm{B} 2$ und $\mathrm{B} 3$ nur geringfügig oberhalb der ermittelten Gesamtreibungszahlen $\mu_{\text {totL }}$ beim Gleiten liegen. Anders ist dies bei der Zinklamellenbasisschicht B1 sowie der Zinknickelbeschichtung B4. Hier liegt die Haftreibungszahl $\mu_{\mathrm{H}}$ weit oberhalb der Gesamtreibungszahl $\mu_{\text {tott }}$ beim Gleiten und führt daher zu einem sogenannten Losbrechen. In wieweit ein solches Verhalten bei Temperaturen von $\vartheta=150^{\circ} \mathrm{C}$ eine generelle zusätzliche Sicherheit gegen selbsttätiges Losdrehen darstellt, ist umstritten und wird daher z.B. in [10] und [5] nicht zur Auswertung des Temperaturverhaltens herangezogen.

Eine Variation der Mutternbeschichtung (P1 und P4: blank; P2 und P3: verzinkt) bei gleich beschichteter Schraube der Abmessung M6, jedoch unterschiedlicher Schraubengeometrie (P1 und P2: Kombischraube; P3 und P4: Außenträger) verdeutlicht auch hier die Abhängigkeit der Gesamtreibungszahlen beim Lösevorgang (Abb. 4). Einzig Prüfgutvariante P4 mit außentragender Schraube und blanker Mutter kann den Warmlöseversuch mit einer gemittelten Gesamtreibungszahl von $\varnothing_{\mu_{\text {totL }}}=0,063$ bestehen.

\section{Vergleich der Prüfmethoden}

Für den Vergleich der Prüfmethoden: Anzieh- und Löseversuche an einem Anziehprüfstand (1) und Warmlöseversuch nach VDA 235-203 [10]/VW 01131 [5] (2) werden die Ergebnisse der Schraubenabmessung M10 herangezogen (Prüfgutvarianten P5, Abb. 5). Es wird ersichtlich, dass die beiden Prüfmethoden für das Verhältnis von Losdreh- zu Anziehdrehmoment $\mathrm{T}_{\mathrm{LG}} / \mathrm{T}$ sowie die Gesamtreibungszahlen

\footnotetext{
${ }^{1}$ Die Haftreibungszahl $\mu_{\mathrm{H}}$ kann beim Lösevorgang aus dem Losbrechmoment $\mathrm{T}_{\mathrm{LH}}$, den Geometriegrößen und der angenommenen Restvorspannkraft bestimmt werden.
}

$\mu_{\text {totL }} \mathrm{zu}$ ähnlichen Ergebnissen führen. Hierbei zeigen sich systematische Abhängigkeiten: Die ermittelten Momentenverhältnisse $T_{\mathrm{LG}} / \mathrm{T}$ und Gesamtreibungszahlen $\mu_{\mathrm{totL}}$ liegen bei Prüfmethode 1 stets oberhalb der Ergebnisse von Prüfmethode 2. Diese Abweichungen können im Versuchsaufbau und der Prüfmethode selbst begründet sein, auch wenn ähnliche Klemmlängenbereiche vorlagen (Prüfmethode 1: $1_{\mathrm{K}} \mathrm{M} 10_{0}=42 \mathrm{~mm}$; Prüfmethode 2: $1_{\mathrm{K}} \mathrm{M} 10=43 \mathrm{~mm}$ ). Bei Prüfmethode 1 ergibt sich gegenüber Prüfmethode 2:

- eine höhere Nachgiebigkeit des MehrkomponentenMesswertaufnehmers als bei dem starren Prüfkörper, so dass es beim Setzen der Schraubenverbindung im Messwertaufnehmer zu einem geringeren Vorspannkraftverlust kommt.

- umgekehrt aber auch eine größere Anzahl an Trennfugen beim Mehrkomponenten-Messwertaufnehmer, die zu einem höheren Vorspannkraftverlust führen können

- eine längere Wartezeit von $\mathrm{t}=4 \mathrm{~h}$ bis zum Erreichen der Solltemperatur von $\vartheta=150^{\circ} \mathrm{C}$ gegenüber einer Wartezeit von $\mathrm{t}=2,5 \mathrm{~h}$ bei Prüfmethode 2, die in Abhängigkeit der Paarung ggf. zu einem höheren Vorspannkraftverlust führen kann

- nicht die Notwendigkeit Annahmen vorzunehmen.

\section{Zusammenfassung}

Die Ergebnisse in diesem Beitrag haben gezeigt, wie stark die Gesamtreibungszahlen $\mu_{\text {tot }}$ beim Anzieh- und Lösevorgang vom tribologischen System der Schraubenverbindung abhängen. Sobald eine Komponente variiert wird, ergeben sich andere Gesamtreibungszahlen $\mu_{\mathrm{tot}}$. Dies kann dazu führen, dass die festgelegte Selbsthemmungsgrenze bei einer Temperatur von $\vartheta=150^{\circ} \mathrm{C}$ unterschritten wird und die Schraubenverbindung nicht den Warmlöseversuch nach VDA 235-203 [10]/VW 01131 [5] besteht. Es lässt sich schlussfolgern, dass in Abhängigkeit der gewählten Schrau- 
benverbindung Warmlöseversuche an Originalbauteilen durchgeführt werden müssen, da ein genauer Übertrag der Ergebnisse von einer Variante zu einer anderen Variante nicht möglich ist.

Weiterhin konnte mit den exemplarisch gewählten Beschichtungen B1-B4 verdeutlicht werden, dass je nach Beschichtungssystem eine mehr oder weniger starke Abhängigkeit der Gesamtreibungszahl von der Temperatur vorliegt. Die Gesamtreibungszahl kann dabei mit zunehmender Temperatur steigen oder fallen. Versuche mit beiden Prüfmethoden haben gezeigt, dass eine große Temperaturabhängigkeit sowohl bei der Zinklamellenbeschichtungen B2 als auch bei der Zinknickelbeschichtungen B4 durch organische Bestandteile gegeben ist. Die zusätzliche Untersuchung der Haftreibungszahl $\mu_{\mathrm{H}}$ lässt erkennen, dass diese ggf. nur dann als weitere Sicherheit gegenüber dem selbsttätigen Losdrehen betrachtet werden kann, wenn sie weit oberhalb der Gesamtreibungszahl beim Lösevorgang $\mu_{\text {totL }}$ liegt. Die Ergebnisse verdeutlichen, dass geringe Gesamtreibungszahlen $\mu_{\mathrm{tot}}$ beim Lösevorgang nicht pauschal auch zu niedrigen Haftreibungszahlen $\mu_{\mathrm{H}}$ führen müssen. Jedoch stellt eine Betrachtung der Gesamtreibungszahlen beim Gleiten ohne die Haftreibungszahlen eine konservative Sichtweise dar.

Eine Gegenüberstellung der beiden Prüfmethoden: Warmlöseversuche nach VDA 235-203 [10]/VW 01131 [5] oder Anzieh- und Löseversuche an einem Anziehprüfstanden zeigen eine grundsätzliche Vergleichbarkeit der Ergebnisse auf. Hierbei sind die Ergebnisse von Prüfmethode 2 stets kritischer bezüglich der Selbsthemmungsgrenze als die von Prüfmethode 1.

\section{Hinweis}

Die in diesem Beitrag mitgeteilten Ergebnisse und Ausführungen beziehen sich ausschließlich auf das exemplarisch ausgewählte Prüfgut sowie die beispielhaft verwendeten Beschichtungen. Andere Spezifikationen von Beschichtungen mit organischer, anorganischer oder silikatischer Deckschicht sowie Versiegelung können sich bezüglich der Temperaturabhängigkeit der Reibungszahlen anders verhalten und bedürfen einer gesonderten Untersuchung.

Förderung Dieser Beitrag wurde von der Firma Walter Hillebrand $\mathrm{GmbH} \& \mathrm{Co}$. KG in Form von Beschichtungen unterstützt, wofür sich die Autoren herzlich bedanken.

Funding Open Access funding enabled and organized by Projekt DEAL.

Interessenkonflikt A. Eberhard, M. Stähler, S. Beyer, M. Klein und M. Oechsner geben an, dass kein Interessenkonflikt besteht.

Open Access Dieser Artikel wird unter der Creative Commons Namensnennung 4.0 International Lizenz veröffentlicht, welche die Nut- zung, Vervielfältigung, Bearbeitung, Verbreitung und Wiedergabe in jeglichem Medium und Format erlaubt, sofern Sie den/die ursprünglichen Autor(en) und die Quelle ordnungsgemäß nennen, einen Link zur Creative Commons Lizenz beifügen und angeben, ob Änderungen vorgenommen wurden.

Die in diesem Artikel enthaltenen Bilder und sonstiges Drittmaterial unterliegen ebenfalls der genannten Creative Commons Lizenz, sofern sich aus der Abbildungslegende nichts anderes ergibt. Sofern das betreffende Material nicht unter der genannten Creative Commons Lizenz steht und die betreffende Handlung nicht nach gesetzlichen Vorschriften erlaubt ist, ist für die oben aufgeführten Weiterverwendungen des Materials die Einwilligung des jeweiligen Rechteinhabers einzuholen.

Weitere Details zur Lizenz entnehmen Sie bitte der Lizenzinformation auf http://creativecommons.org/licenses/by/4.0/deed.de.

\section{Literatur}

1. Verband der Automobilindustrie e. V. (2009) VDA 235-101 VDAPrüfblatt Reibungszahleinstellung von mechanischen Verbindungselementen mit metrischem Gewinde. Verband der Automobilindustrie e. V., Frankfurt

2. BMW Group Standard (2010) GS 90010-1 Oberflächenschutzarten für metallische Werkstoffe

3. Mercedes-Benz (2012) DBL 9440 Verbindungselemente mit metrischem Gewinde Oberflächenschutz und ergänzende Technische Lieferbedingungen

4. Volkswagen (2012) VW 01129 Grenzwerte der Reibungszahlen

5. Volkswagen Aktiengesellschaft (2018) VW 01131 Ermittlung von Reibungszahlen. Volkswagen Aktiengesellschaft, Wolfsburg

6. Sommer K, Heinz R, Schöfer J (2010) Verschleiß metallischer Werkstoffe, Erscheinungsformen sicher beurteilen, 1. Aufl. Vieweg + Teubner Verlag, Wiesbaden

7. DIN Deutsches Institut für Normung e. V. (2020) DIN 969 Verbindungselemente mit Gewinde - Schwingfestigkeitsversuch bei Axialbelastung - Prüfverfahren und Auswertung der Ergebnisse. Beuth, Berlin

8. DIN Deutsches Institut für Normung e. V. (2002) DIN 65151 Luftund Raumfahrt, Dynamische Prüfung des Sicherungsverhaltens von Schraubverbindungen unter Querbeanspruchung (Vibrationsprüfung). Beuth, Berlin

9. DIN Deutsches Institut für Normung e.V. (2013) DIN EN ISO 16047 Verbindungselemente - Drehmoment / Vorspannkraft-Versuch. Beuth, Berlin

10. Verband der Automobilindustrie e. V. (2005) VDA 235-203 VDAPrüfblatt Verschraubungsverhalten / Reibungszahlen; Praxis- und montageorientierte Prüfung. Verband der Automobilindustrie e. V., Frankfurt

11. DIN Deutsches Institut für Normung e. V. (2013) DIN EN ISO 4032 Sechskantmuttern Typ 1 - Produktklassen A und B. Beuth, Berlin

12. Volkswagen Aktiengesellschaft (2007) VW 60449 VierkantSchweißmutter Hohe Ausführung. Volkswagen Aktiengesellschaft, Wolfsburg

13. Ford Global Technologies, LLC (2004) WA 100 bolts - hexagon flange head fully threaded. Ford Global Technologies, LLC, Dearborn

14. Födisch F, Hörnig T, Johne V, Friedrich C, Füssel U (2017) Maßnahmen zur Vermeidung von Stick-Slip-Ereignissen in der Schraubmontage. In: 10. Workshop Industrielle Schraubmontage. Deutscher Schraubenverband e. V., Dresden

15. Eberhard A, Stähler M, Beyer S, Beyer J, Oechsner M (2017) Anzieh- und Löseverhalten von Schraubenverbindungen unter Temperatur bei unterschiedlicher Versuchsmethodik. In: 12. Workshop Industrielle Schraubmontage. Deutscher Schraubenverband e. V., Dresden 